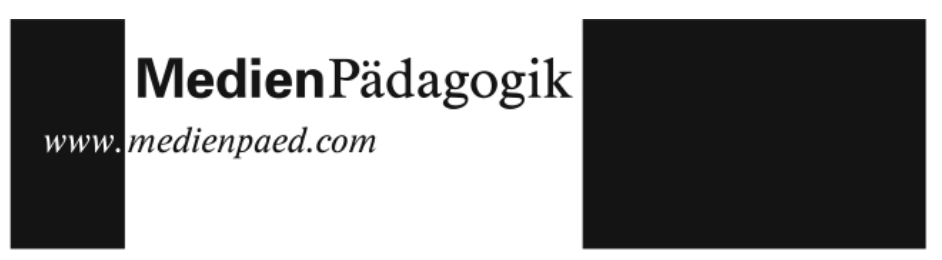

Rezensionen

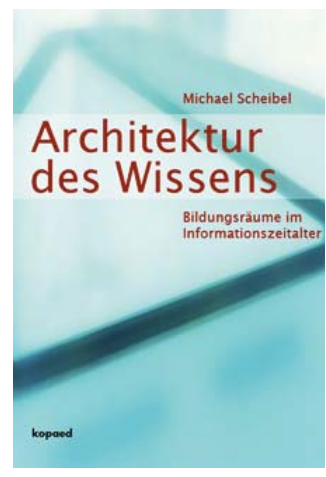

Michael Scheibel

\title{
Architektur des Wissens.
}

Bildungsräume im Informationszeitalter.

München: kopaed, 2008. 190 Seiten

ISBN-13 978-3-86736-028-9

$€ 14.80 ;$ CHF 23.40

Wer braucht noch Computerräume? Über wissensarchitektonische Möglichkeiten der Gestaltung von Lernräumen.

Daniela Küllertz

Die Entstehung von Wissens-, Lern- und Bildungsräumen durch neue Kommunikationstechnologien und Medien wurde im Fachgebiet Medienbildung und in der Medienpädagogik sowohl durch die Beschäftigung mit Möglichkeiten und Grenzen des E-Learnings, besonders aber durch so genannte Web2.0-Phänomene wie dem semantischen Web zu einem wichtigen Gegenstand. Michael Scheibel präsentiert in seiner Monografie «Architektur des Wissens. Bildungsräume im Informationszeitalter.» ein erfrischend originell aufgebautes Bild des Verhältnisses von Kommunikationstechnologien als auch -medien und der Entstehung und Veränderung von Wissensräumen. Das Buch ist für ein akademisches Publikum wegen der fehlenden strukturierten Argumentation, der szenischen Erzählweise und dem assoziativen Charakter der Buchkapitel und Unterkapitel eher ungewohnt und wohl an einen breiten Kreis am Thema interessierter Leser gerichtet. Wie die Wortwolken-Darstellung (flächige Schlagworthaufen) des Inhaltsverzeichnisses ist die Form des gesamten Buch dem thematisierten Inhalt, der Darstellung dezentralisierter, nicht-hierarchischer und verdichteter Wissens- und Kommunikationsräume angepasst.

\section{Vernetzung, Beschleunigung und Dezentralisierung von Wissensräu- men}

Der Künstler, Kurator, Journalist und Wissenschaftler Scheibel stellt einführend fest, dass der bestehende Bildungsraum mit seinen Lehr- und Lernmedien einem allmählichen Wandel unterliege, den es im Hinblick auf die Entstehung neuer Wissensräume mit zu gestalten gilt. Es folgt mit der Darstellung der Warburg-Bibliothek als technisierter und rationalisierter Verknüp- 
fung bildhaften und technischen Wissens, mit der Radiotheorie Brechts und dem Electronic Super Highway des Medienkünstlers Nam June Paik eine erste beispielhafte Betrachtung des Themenbereiches. Scheibel thematisiert den Einfluss von Technologien und Medien auf veränderte Raumwahrnehmung. So ziele die Fotografie auf «die gleichzeitige Anwesenheit aller Vergangenheit in der Gegenwart», im «Gegenwartsfenster der Monitore» erscheinen beschleunigt Daten, die ebenso beschleunigt in die „Latenz der Speicher» absinken, um von dort sofort wieder aufzutauchen (S. 28f). Die Welt verdichtet sich zu prägnanten Punkten und diese Punkte erweitern sich zu Raumfeldern, so der Autor. Anknüpfend an den Medientheoretiker de Kerckhove bezeichnet Scheibel die Entstehung eines neuen Raumkonzeptes durch die vernetzende Perspektive. Dem Raum werde «eine neue virtuelle Erweiterung hinzugefügt und den Wänden eine neue Eigenschaft als Schnittstellen» (S. 43). Dabei stehe der Mensch als navigierende Verknüpfung inmitten der Darstellung von bestenfalls fragmentarischem und dezentralisiertem Wissen. Im Informationszeitalter sei die Zentralperspektive als erkenntnistheoretische und pädagogische Grundhaltung obsolet, gefordert seien Fähigkeiten wie Selbstorganisation und Selbstkontrolle.

\section{Partizipative Lernformen in veränderten Wissensräumen}

Das zweite Kapitel wird durch ein Zitat des Medientheoretikers Giesecke eingeleitet, in welchem für die Bildungspolitik posttypografische Wissensund Kommunikationskonzepte gefordert werden. Danach folgt unvermittelt eine Beschreibung der von Flusser vorgenommenen Unterscheidung diskursiver und dialogischer Kommunikationsmedien, deren Bedeutung für Scheibel in der Berücksichtigung des Stellenwertes von Räumen und Technologien für Kommunikationsformen liegt. Es folgen mit der Beschreibung der Reformuniversität Bielefeld und der Fernuniversität Hagen Beispiele für Kommunikations- und Wissensarchitekturen, denen Interviews mit dem Architekten Klaus Köpke und dem Gründungsrektor der Fernuniversität Otto Peters zur Seite gestellt werden. Während die Architektur der Reformuniversität Bielefeld baulich die Leitgedanken einer an Verwissenschaftlichungsprozesse anzupassenden Universität durch «Vernetzung und Kommunikation, Rationalisierung und Variabilität» (S. 58) vollende, habe die Fernuniversität Hagen das Modell einer Fernlehre unter Auslotung der medientechnologischen Möglichkeiten entwickelt und dabei auf andere didaktische Konzepte wie «eigenständige, partizipative und verstärkt prozesshaft bestimmte Handlungs- und Lernformen» (S. 71) zurückgegriffen. Scheibel referiert 
schließlich unter dem tag «Transformierung» die historische Rekonstruktion europäischer Visionen der Informationsgesellschaft von Giesecke.

\section{Orientierung in beweglichen Wissensräumen}

Das dritte Kapitel thematisiert die Raummetaphorik des Internet, das in den 90er Jahren als utopischer Ort des Experimentierens verstanden wurde. Virtuelle Räume wie die digitale Stadt wurden zum «Bedeutungsträger für revolutionierte kommunikative und soziale Strukturen» (S. 90). Anknüpfend an die Arbeiten der Soziologin Löw betont Scheibel die Bedeutung von Kommunikationstechnologien und beweglich gewordenen Räumen für ein verändertes nicht-euklidisches Raumverständnis. Im Interview mit Monika Fleischmann und Wolfgang Strauß ist Beweglichkeit des vernetzten Datenraumes als Wissensraum die zentrale Herausforderung des Internets. Scheibel stellt die Plattform netzspannung.org als Wissensraum vor, für die Fleischmann und Strauß so genannte Knowledge Discovery Tools wie die semantic map entwickeln. Die Wissensgenerierung in Netzwerken bedeutet für Scheibel, dessen „Positionierung» eher implizit deutlich wird, zugleich Transformation von Bildungsinstitutionen. Eine solche Transformation stellen die Faßlerschen «Communities of Projects» als «weltweit vernetzte, episodische Momentgemeinschaften» (2008: 110) dar. Ob diese vorgeblich nichtinstitutionellen, durch Programme regulierten Handlungsräume der «Communities of Projects» eine Krise der Institution darstellen, ist jedoch fraglich, wenn man sich Scheibels Beispiel netzspannung.org als institutionelles Produkt vor Augen führt.

\section{Schnittstellen zwischen Lernort und Wissensraum}

Im vierten und letzten Abschnitt des Buches thematisiert Scheibel die Beziehung virtueller Räume mit physischen Umgebungen. Am Beispiel des Projektes Roomware ${ }^{\circledR}$ des Forschungsbereiches AMBIENTE des Fraunhofer Instituts IPSI greift Scheibel die Konstruktion neuer Schnittstellen zwischen physischen und virtuellen Raum auf. Mit der Darstellung der HochschulInfostruktur der ETH World, einem Planungskonzept zur Umstrukturierung der Eidgenössischen Technischen Hochschule Zürich, schließt Scheibel seine Ausführungen über Wissensarchitekturen und Bildungsräume im Informationszeitalter ab. In dieser Infostruktur stehe der Mensch im Zentrum inm dienender Technologien, so der interviewte Informationsarchitekt Gerhard Schmitt. Tradierte Computerräume sind obsolet, es gelte eine Informationsarchitektur auf der Höhe technischer Möglichkeiten zu schaffen. Ab- 


\section{Rezensionen}

schließend wird der Informationspädagoge Werner Sesink zitiert, der davon spricht, dass «under construction» die neue Grundverfassung von Bildungseinrichtungen werde, die als Chance für offene Strukturen, Kreativität und «permanente Meta-Reflexion" angesehen werden müsse.

Scheibels Argumentation, dass die Bildungspolitik Konzepte braucht, die den Eigenschaften und Möglichkeiten von Digitalisierung und Vernetzung angemessen sind, ist nachvollziehbar. Die Frage ist, ob architektonisches Denken gewinnbringende Konzepte inspirieren kann. Scheibel liefert nach meiner Auffassung interessante Einblicke in avantgardistisch-künstlerische Informationsraumgestaltung. Er wirft Schlaglichter auf das ästhetische Ausloten und Gestalten neuer Wissensräume, die er offenbar zugleich als Bildungsräume ansieht. Die Gleichsetzung von institutionalisierten Lehr- und Lernräumen wie (Fern-)Universitäten und Schulen mit Bildung ist im Hinblick die bildungstheoretische Diskussion fragwürdig. Eine reflektierte Verwendung des Bildungsbegriffes hätte dazu beigetragen, die medienbildungstheoretische Diskussion noch stärker raumtheoretisch zu inspirieren. Zudem fordert die starke medienwissenschaftliche Orientierung des Autors stellenweise Tribut in Kurzschlüssen von der Ebene der Kommunikationsbedingungen auf die Ebene sozialer Wirklichkeit. So ist es etwa fraglich, ob medienwissenschaftlich inspirierte Zeitdiagnosen wie die Annahme einer Welt des Informationszeitalters, «die keine Fixpunkte und Konstanten mehr zu bieten scheint» (S. 36), sozialwissenschaftlich haltbar sind.

Wenngleich die teilweise unvermittelt erscheinende Abfolge der Abschnitte und Themen den (akademisch sozialisierten) Leser manchmal etwas ratlos erscheinen lassen, handelt es sich um eine originelle und interessante Darstellung möglicher und verwirklichter Informationsraumarchitekturen und Wissensraumkonstruktionen. Insgesamt ist das Buch lesenswert. 